\title{
A FURTHER EXTENSION OF BANACH'S CONTRACTION PRINCIPLE
}

\author{
M. MARJANOVIĆ
}

1. Introduction. M. Edelstein, in [1], has extended the well-known Banach's contraction principle introducing the notion of $(\epsilon, \lambda)$ uniformly locally contractive mapping, i.e., such a mapping $f: M \rightarrow M$ of a metric space $M$ into $M$ that

$$
p, q \in S(x, \epsilon) \Rightarrow d(f p, f q)<\lambda d(p, q)
$$

for every $x \in M$, where $\epsilon$ and $\lambda(0 \leqq \lambda<1)$ do not depend on $x$. The space $M$ is $\epsilon$-chainable if for every two $a, b \in M$ there is a sequence of elements in $M: x_{0}, x_{1}, \cdots, x_{n}$ such that $x_{0}=a, x_{n}=b$ and $d\left(x_{i}, x_{i+1}\right)<\epsilon, i=0,1, \cdots, n-1$. So, the Edelstein's extension reads: If $M$ is a complete, $\epsilon$-chainable metric space and $f: M \rightarrow M$ is an $(\epsilon, \lambda)$-uniformly locally contractive mapping then there exists a unique point $\xi \in M$ such that $f \xi=\xi$.

S. Prešić, in [2], (see also [3]), has considered a mapping $f: M^{n} \rightarrow M$ such that

$$
\begin{gathered}
d\left(f\left(x_{1}, x_{2}, \cdots, x_{n}\right), f\left(x_{2}, x_{3}, \cdots, x_{n+1}\right)\right) \\
\leqq q_{1} d\left(x_{1}, x_{2}\right)+q_{2} d\left(x_{2}, x_{3}\right)+\cdots+q_{n} d\left(x_{n}, x_{n+1}\right)
\end{gathered}
$$

where $q_{i} \geqq 0$ and $q_{1}+q_{2}+\cdots+q_{n}<1$ and the numbers $q_{i}$, in (2), do not depend on $x_{i}$. He proves that the sequence

$$
x_{8+n}=f\left(x_{8}, x_{8+1}, \cdots, x_{8+n-1}\right)
$$

converges to a unique point $\xi \in M$, such that $\xi=f(\xi, \xi, \cdots, \xi)$.

It seems natural to try to give a nonmetric form to the notion of $\epsilon$-chainable space and so extend further the result from [1] and it is interesting to try to obtain the iterates from [2] as usual ones in the proof of Banach's contraction principle. Both of these will be the aim of this paper.

2. Notations and definitions. In order to explain some of our notations, we will repeat the definition of a metric space. Let $M$ be a nonempty set, $M^{2}=M \times M$ its direct product. A point from $M$ is denoted by a small and a point from $M^{2}$ by a capital letter, i.e., $x \in M$ and $X=\left(x^{\prime}, x^{\prime \prime}\right) \in M^{2}$. A metric on $M$ is a real-valued function $\|\cdot\|: M^{2} \rightarrow[0,+\infty)$ such that

1. $\|x\|=0 \Leftrightarrow p R_{1} X=p R_{2} X$ (i.e., $x^{\prime}=x^{\prime \prime}$ ),

Received by the editors December 29, 1966. 
2. $\|X\|=\left\|X^{-1}\right\|$, where $X^{-1}=\left(x^{\prime \prime}, x^{\prime}\right)$,

3. $\left\|X_{1} \circ X_{2}\right\| \leqq\left\|X_{1}\right\|+\left\|X_{2}\right\|$, where $X_{1} \circ X_{2}$ is the composition of pairs.

Instead of $\|X\|$ we will also write $d\left(x^{\prime}, x^{\prime \prime}\right)$ and the pair $(M, d)$ is a metric space with the metric $d$. To each mapping $f: M \rightarrow M$ we correspond a new one $F: M^{2} \rightarrow M^{2}$ such that $F X=\left(f x^{\prime}, f x^{\prime \prime}\right)$.

Definition 1. A metric space $M$ is $U$-chainable, where $U \subset M^{2}$ if

$$
M^{2}=U\left\{U^{n}: n \in N\right\}, \quad U^{n}=U \circ U \circ \cdots \circ U \text { (ntimes) }
$$

Definition 2. A mapping $f: M \rightarrow M$ is a $(q, k)$-contraction if for every $X \in M^{2}$

$$
\left\|F^{k} X\right\| \leqq q \cdot \operatorname{Max}\left\{\left\|F^{i} X\right\|: i=0,1, \cdots, k-1\right\},
$$

where $0 \leqq q<1$ and $F^{k} X=F F^{k-1} X$.

It is easily seen that each contraction (i.e., $(q, 1)$-contraction) is $(q, k)$-contraction and that $\left\|F^{n} X\right\| \leqq q^{s} a_{X}$, where $a_{X}=\operatorname{Max}\left\{\left\|F^{i} X\right\|\right.$; $i=0,1, \cdots, k-1\}$ and $S=[n / k]$ ( $s$ is the greatest integer not exceeding $n / k$ ).

Definition 3. A mapping $f: M \rightarrow M$ is $(U, q, k)$-contraction if for every $X \in U$

$$
\left\|F^{k} X\right\| \leqq q \cdot \operatorname{Max}\left\{\left\|F^{i} X\right\|: i=0,1, \cdots, k-1\right\},
$$

where $0 \leqq q<1$ and $F U \subseteq U$.

Now, we are going to prove a theorem which contains the results mentioned above ([1] and [2]) as special cases.

THEOREM. Let $f: M \rightarrow M$ be a continuous mapping of a complete metric space into itself which is a $(U, q, k)$-contraction. If $M_{0}^{2}$ $=U\left\{U^{n}: n \in N\right\}$ and $M \times f M \subseteq M_{0}^{2}$, then all sequences of iterates $x_{n}=f^{n}\left(x_{0}\right), x_{0} \in M$ converge to a fixed point of $f$. When $M$ is $U$-chainable $f$ has a unique fixed point.

Proof. For an arbitrary $X_{0} \in M_{0}^{2}$ there is $r \in N$ such that

$$
X_{0}=Y_{1} \circ Y_{2} \circ \cdots \circ Y_{r} ; \quad Y_{i} \in U, i=1,2, \cdots, r .
$$

Let

$$
X_{n+1}=F X_{n}, \quad n=0,1,2, \cdots,
$$

then

$$
\begin{aligned}
\left\|X_{n}\right\| & \leqq\left\|F^{n} Y_{1}\right\|+\left\|F^{n} Y_{2}\right\|+\cdots+\left\|F^{n} Y_{r}\right\| \\
& \leqq q^{S} \cdot a_{Y_{1}}+q^{S} \cdot a_{Y_{2}}+\cdots+q^{S} \cdot a_{Y_{r}}=q^{S} \cdot a,
\end{aligned}
$$

where

$$
a=\sum\left\{a_{Y_{i}}: i=1,2, \cdots, r\right\} .
$$


Since $M \times f M \subseteq M_{0}^{2}$ for each $x_{0} \in M,\left(x_{0}, f x_{0}\right) \in M_{0}^{2}$, so that $\left\|F^{n} X_{0}\right\|$ $\leqq q^{s} \cdot a, X_{0}=\left(x_{0}, f x_{0}\right)$. This means that the sequence $x_{n}=f^{n} x_{0}$ is Cauchy convergent and $M$ being complete it converges to some $\tilde{x} \in M$. From the continuity of $f$ it follows that $\tilde{x}$ is a fixed point of $f$.

In the case when $M$ is $U$-chainable, for $X_{0}=\left(x_{0}^{\prime}, x_{0}^{\prime \prime}\right)$

$$
\left\|X_{n}\right\| \leqq q^{s} \cdot a \rightarrow 0, \quad n \rightarrow \infty,
$$

so that both sequences $x_{n}^{\prime}=f^{n}\left(x_{0}^{\prime}\right)$ and $x_{n}^{\prime \prime}=f^{n}\left(x_{0}^{\prime \prime}\right)$ converge to the same point $\tilde{x}$. Hence $\tilde{x}$ is unique.

EXAmple 1. Taking $U=\{X:\|X\|<\epsilon\}$ and $k=1$, we get the result in $[1]$.

ExAmple 2. Let $f: M^{2} \rightarrow M$ and let $\bar{f}: M^{2} \rightarrow M^{2}$ be such that

$$
\tilde{f}\left(x^{\prime}, x^{\prime \prime}\right)=\left(x^{\prime \prime}, f\left(x^{\prime}, x^{\prime \prime}\right)\right) \text {. }
$$

Choosing $U$ to be

$$
U=\left\{\left(x^{\prime}, x^{\prime \prime}, x^{\prime \prime}, f\left(x^{\prime}, x^{\prime \prime}\right)\right):\left(x^{\prime}, x^{\prime \prime}\right) \in M^{2}\right\}
$$

and supposing, as it has been in [2], that

$$
d\left(f\left(x^{\prime}, x^{\prime \prime}\right), f\left(x^{\prime \prime}, x^{\prime \prime \prime}\right)\right) \leqq q_{1} d\left(x^{\prime}, x^{\prime \prime}\right)+q_{2} d\left(x^{\prime \prime}, x^{\prime \prime \prime}\right)
$$

and introducing a metric $\delta$ on $M^{2}$ such that

$$
\delta\left[\left(x_{1}^{\prime}, x_{1}^{\prime \prime}\right),\left(x_{2}^{\prime}, x_{2}^{\prime \prime}\right)\right]=\operatorname{Max}\left\{d\left(x_{1}^{\prime}, x_{2}^{\prime}\right), d\left(x_{1}^{\prime \prime}, x_{2}^{\prime \prime}\right)\right\}
$$

then $\bar{f}$ is a $(U, q, 2)$-contraction, where $q_{1}+q_{2} \leqq q<1$ and $q_{1}$ and $q_{2}$ need not be independent of $x^{\prime}, x^{\prime \prime}, x^{\prime \prime \prime}$. Let us write for short

$$
x^{\prime \prime \prime}=f\left(x^{\prime}, x^{\prime \prime}\right), \quad x^{\mathrm{IV}}=f\left(x^{\prime \prime}, x^{\prime \prime \prime}\right), \quad x^{\mathrm{v}}=f\left(x^{\prime \prime \prime}, x^{\mathrm{IV}}\right) .
$$

Then,

$$
\begin{aligned}
\tilde{f}\left(x^{\prime}, x^{\prime \prime}\right) & =\left(x^{\prime \prime}, x^{\prime \prime \prime}\right), & \tilde{f}^{2}\left(x^{\prime}, x^{\prime \prime}\right)=\left(x^{\prime \prime \prime}, x^{\mathrm{Iv}}\right), \\
\tilde{f}\left(x^{\prime \prime}, x^{\prime \prime \prime}\right) & =\left(x^{\prime \prime \prime}, x^{\mathrm{Iv}}\right), & \tilde{f}^{2}\left(x^{\prime \prime}, x^{\prime \prime \prime}\right)=\left(x^{\mathrm{Iv}}, x^{\mathrm{v}}\right)
\end{aligned}
$$

and it is easily checked that

$$
\begin{gathered}
\quad \delta\left[\tilde{f}^{2}\left(x^{\prime}, x^{\prime \prime}\right), \tilde{f}^{2}\left(x^{\prime \prime}, x^{\prime \prime \prime}\right)\right] \\
=\operatorname{Max}\left\{d\left(x^{\prime \prime \prime}, x^{\mathrm{Iv}}\right), d\left(x^{\mathrm{Iv}}, x^{\mathrm{v}}\right)\right\} \\
\leqq q \cdot \operatorname{Max}\left\{\delta\left[\left(x^{\prime}, x^{\prime \prime}\right),\left(x^{\prime \prime}, x^{\prime \prime \prime}\right)\right], \delta\left[\tilde{f}\left(x^{\prime}, x^{\prime \prime}\right), \tilde{f}\left(x^{\prime \prime}, x^{\prime \prime \prime}\right)\right]\right\},
\end{gathered}
$$

which proves that $\tilde{f}$ is a $(U, q, 2)$-contraction. Iterating the mapping $\bar{f}$ in the usual way and starting with the point $\left(x_{0}, x_{1}\right)$ we get

$$
\begin{aligned}
& \tilde{f}\left(x_{0}, x_{1}\right)=\left(x_{1}, f\left(x_{0}, x_{1}\right)\right)=\left(x_{1}, x_{2}\right), \\
& \tilde{f}\left(x_{1}, x_{2}\right)=\left(x_{2}, f\left(x_{1}, x_{2}\right)\right)=\left(x_{2}, x_{3}\right)
\end{aligned}
$$


and so on. Since the topology induced by the metric $\delta$ is identical to the product topology, the sequence $\left\{x_{n}\right\}$ is convergent to the point $\tilde{x}$ and $\tilde{x}=f(\tilde{x}, \tilde{x})$. The continuity of $f$ and the uniqueness of $\tilde{x}$ are proved easily as it has been done in [2]. The case $f: M^{n} \rightarrow M$ is treated analogously.

Note that in [2] a sequence of mappings has been considered but under more restrictive conditions.

EXAMPLE 3. We are giving here an example of a mapping which is not a uniformly local contraction in the sense of [1] and which still satisfies the conditions of the theorem proved here and so has a unique fixed point.

Let

$$
\begin{aligned}
K_{n} & =\left\{(x, y):\left(x-\left(2^{n}-1\right) / 2^{n}\right)^{2}+y^{2}=\left(1 / 2^{n}\right)^{2}\right\}, \\
L_{n} & =\left\{(x, y):(x, y) \in K_{n}, x>\left(2^{n}-1\right) / 2^{n}, y>0\right\}
\end{aligned}
$$

and let $S_{n}=K_{n} \backslash L_{n}$. If a mapping $f$ is defined on $S=U\left\{S_{n}: n \in N\right\}$ such that each point $A \in S_{n}$ at the arc distance $s$ from the point $(1,0)$ is mapped on the point $f(A) \in S_{n}$ at the arc distance $s / 2$ from $(1,0)$, then $f$ is an $\epsilon_{n}$-uniformly local contraction on $S_{n}$ (see [1]). Let

$$
U=\left\{\left(A, A^{\prime}\right): A, A^{\prime} \in S_{n} \text { and } d\left(A, A^{\prime}\right)<\epsilon_{n}\right\} .
$$

Then $S$ is $U$-chainable and $f$ is a $(U, q, 1)$-contraction so that, $S$ being complete, $f$ has a unique fixed point. (It is the point $(1,0)$.)

\section{REFERENCES}

1. M. Edelstein, An extension of Banach's contraction principle, Proc. Amer. Math. Soc. 12 (1961), 7-10.

2. S. Prešić, Sur la convergence des suites, C. R. Acad. Sci. Paris 260 (1965), 38283830.

3. M. Marjanović and S. Prešić, Remark on the convergence of a sequence, Publ. Elekt. Fak. 155 (1965), 63-64.

Institut Mathématique, Belgrade, Yugoslavia 